\title{
Comparison of a Triple Inverted Pendulum Stabilization using Optimal Control Technique
}

\author{
Mustefa Jibril ${ }^{1}$, Messay Tadese ${ }^{2}$, Eliyas Alemayehu Tadese ${ }^{3}$ \\ ${ }^{1}$ Msc, School of Electrical \& Computer Engineering, Dire Dawa Institute of Technology, Dire Dawa, \\ Ethiopia \\ ${ }^{2}$ Msc, School of Electrical \& Computer Engineering, Dire Dawa Institute of Technology, Dire Dawa, \\ Ethiopia \\ ${ }^{3}$ Msc, Faculty of Electrical \& Computer Engineering, Jimma Institute of Technology, Jimma, Ethiopia
}

\begin{abstract}
In this paper, modelling design and analysis of a triple inverted pendulum have been done using Matlab/Script toolbox. Since a triple inverted pendulum is highly nonlinear, strongly unstable without using feedback control system. In this paper an optimal control method means a linear quadratic regulator and pole placement controllers are used to stabilize the triple inverted pendulum upside. The impulse response simulation of the open loop system shows us that the pendulum is unstable. The comparison of the closed loop impulse response simulation of the pendulum with LQR and pole placement controllers results that both controllers have stabilized the system but the pendulum with LQR controllers have a high overshoot with long settling time than the pendulum with pole placement controller. Finally the comparison results prove that the pendulum with pole placement controller improve the stability of the system.
\end{abstract}

Keywords: Inverted pendulum, linear quadratic regulator, Pole placement.

\section{Introduction}

An inverted pendulum is a pendulum that has its center of mass above its pivot point. It is unstable and without additional assist will fall over. It may be suspended stably in this inverted position by means of the usage of a feedback control system to reveal the angle of the pole and flow the pivot factor horizontally returned beneath the center of mass while it begins to fall over, retaining it balanced. The inverted pendulum is a classic problem in dynamics and manage idea and is used as a benchmark for testing control techniques. An inverted pendulum is inherently unstable, and have to be actively balanced with a view to stay upright; this could be accomplished either by applying a torque at the pivot factor, with the aid of transferring the pivot point horizontally as a part of a feedback system, changing the state of rotation of a mass installed at the pendulum on an axis parallel to the pivot axis and thereby generating an internal torque at the pendulum, or with the aid of oscillating the pivot factor vertically. In order to stabilize a pendulum in this inverted position, a feedback control system may be used, which monitors the pendulum's attitude and actions the position of the pivot point sideways while the pendulum starts off evolved to fall over, to hold it balanced.

\section{Mathematical Modeling}

The pendulum consists of three arms that are hinged by ball bearings and can rotate in the vertical plane. The torques $\mathrm{T} 1$ and $\mathrm{T} 2$ are the 
inputs to the pendulum with the middle hinge made free for rotation. By controlling the angles of the arms around specified values, the pendulum can be stabilized inversely with the desired angle attitudes. The triple inverted pendulum is shown in Figure 1 below.

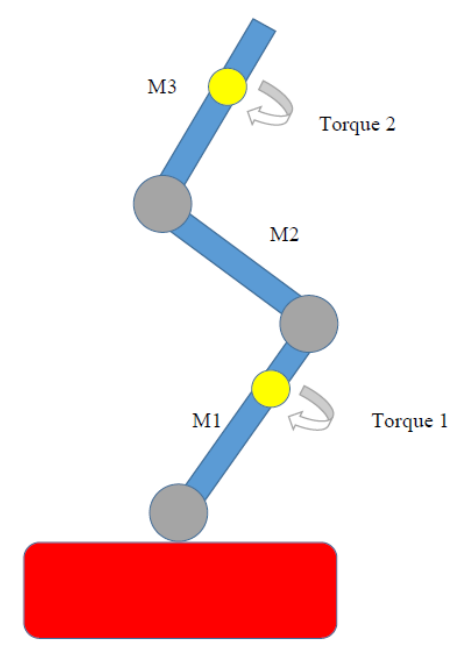

Figure 1 the triple pendulum

Let $\Theta i$ denote the angle of the ith arm measured from the vertical axis as shown in Figure 2 below.
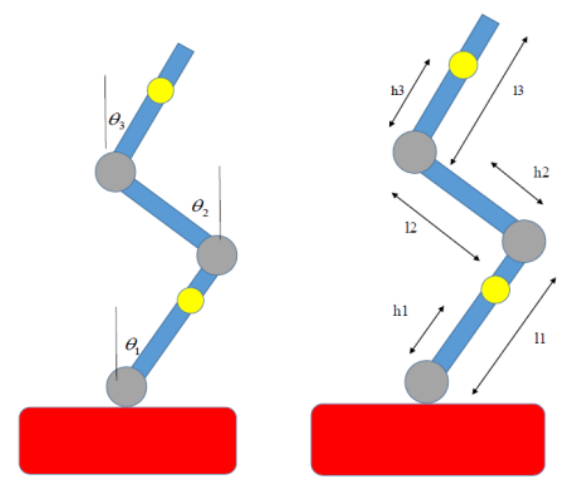

Figure 2 System Configuration

The mathematical modelling of the triple inverted pendulum is derived under the assumption that each arm is a rigid body

Lagrange differential equations is the method used to construct the triple pendulum with a nonlinear vector-matrix differential equation of the form:

$$
\begin{aligned}
& M(\theta) \ddot{\theta}_{i}+N \dot{\theta}_{i}+q_{i}=G T_{j} \\
& i=1,2,3 \\
& j=1,2
\end{aligned}
$$

Where

$$
M(\theta)=\left(\begin{array}{ccc}
J_{1} & l_{1} M_{2} \cos \left(\theta_{1}-\theta_{2}\right) & l_{1} M_{3} \cos \left(\theta_{1}-\theta_{3}\right) \\
l_{1} M_{2} \cos \left(\theta_{1}-\theta_{2}\right) & J_{2} & l_{2} M_{3} \cos \left(\theta_{2}-\theta_{3}\right) \\
l_{1} M_{3} \cos \left(\theta_{1}-\theta_{3}\right) & l_{2} M_{3} \cos \left(\theta_{2}-\theta_{3}\right) & J_{3}
\end{array}\right)
$$

Where

$$
\begin{array}{lll}
M_{1}=m_{1} h_{1}+m_{2} l_{1}+m_{3} l_{1} & \text { and } & J_{1}=I_{1}+m_{1} h_{1}^{2}+m_{2} l_{1}^{2}+m_{3} l_{1}^{2} \\
M_{2}=m_{2} h_{2}+m_{3} l_{2} & & J_{2}=I_{2}+m_{2} h_{2}^{2}+m_{3} l_{2}^{2} \\
M_{3}=m_{3} h_{3} & J_{3}=I_{3}+m_{3} h_{3}^{2}
\end{array}
$$

The $\mathrm{N}$ matrix become:

$$
N=\left(\begin{array}{ccc}
C_{1}+C_{2} & -C_{2} & 0 \\
-C_{2} & C_{2}+C_{3} & -C_{3} \\
0 & -C_{3} & C_{3}
\end{array}\right)
$$

And the $q$ matrix and $\mathrm{G}$ matrix are

$$
\begin{gathered}
q_{1}=l_{1} M_{2} \sin \left(\theta_{1}-\theta_{2}\right) \dot{\theta}_{2}^{2}+l_{1} M_{3} \sin \left(\theta_{1}-\theta_{3}\right) \dot{\theta}_{3}^{2}-M_{1} g \sin \left(\theta_{1}\right) \\
q_{2}=l_{1} M_{2} \sin \left(\theta_{1}-\theta_{2}\right) \dot{\theta}_{1}^{2}+l_{2} M_{3} \sin \left(\theta_{2}-\theta_{3}\right) \dot{\theta}_{3}^{2}-M_{2} g \sin \left(\theta_{2}\right) \\
q_{3}=l_{1} M_{3} \sin \left(\theta_{1}-\theta_{3}\right)\left(\dot{\theta}_{1}^{2}-2 \dot{\theta}_{1} \dot{\theta}_{3}\right)+l_{2} M_{3} \sin \left(\theta_{2}-\theta_{3}\right)\left(\dot{\theta}_{2}^{2}-2 \dot{\theta}_{2} \dot{\theta}_{3}\right)-M_{3} g \sin \left(\theta_{3}\right) \\
G=\left(\begin{array}{cc}
1 & 0 \\
-1 & 1 \\
0 & -1
\end{array}\right)
\end{gathered}
$$

The description of the system is shown in Table 1 below

\begin{tabular}{|l|l|l|}
\hline No & Symbol & Description \\
\hline 1 & $l_{i}$ & length of the ith arm \\
\hline 2 & $h_{i}$ & $\begin{array}{l}\text { the distance from the } \\
\text { bottom to the centre of } \\
\text { gravity } \\
\text { of the } i \text { th arm }\end{array}$ \\
\hline 3 & $m_{i}$ & mass of the ith arm \\
\hline 4 & $\theta_{i}$ & $\begin{array}{l}\text { angle of the ith arm from } \\
\text { vertical line }\end{array}$ \\
\hline
\end{tabular}




\begin{tabular}{|l|l|l|}
\hline 5 & $C_{i}$ & $\begin{array}{l}\text { coefficient of viscous } \\
\text { friction of the ith hinge }\end{array}$ \\
\hline 6 & $I_{i}$ & $\begin{array}{l}\text { moment of inertia of the } \\
\text { i-th arm around the } \\
\text { centre of } \\
\text { gravity }\end{array}$ \\
\hline 7 & $T_{j}$ & $\begin{array}{l}\text { control torque of the jth } \\
\text { hinge }\end{array}$ \\
\hline
\end{tabular}

After linearization of Equation (2) under the assumptions of small deviations of the pendulum from the vertical position and of small velocities, one obtains the following equation

$$
\begin{aligned}
& M \ddot{\theta}_{i}+N \dot{\theta}_{i}+P \theta_{i}=G T_{m_{j}} \\
& i=1,2,3 \\
& j=1,2
\end{aligned}
$$

Where

$$
M=\left(\begin{array}{ccc}
J_{1} & l_{1} M_{2} & l_{1} M_{3} \\
l_{1} M_{2} & J_{2} & l_{2} M_{3} \\
l_{1} M_{3} & l_{2} M_{3} & J_{3}
\end{array}\right)
$$

And

$$
P=\left(\begin{array}{ccc}
M_{1} g & 0 & 0 \\
0 & -M_{2} g & 0 \\
0 & 0 & -M_{3} g
\end{array}\right)
$$

The block-diagram of the pendulum system is shown in Figure 3 and the nominal values of the parameters are given in Table 2.

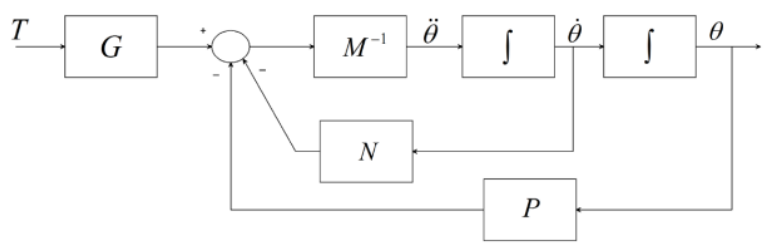

\begin{tabular}{|c|c|c|}
\hline 1 & $h_{1}$ & $0.45 \mathrm{~m}$ \\
\hline 2 & $h_{2}$ & $0.2 \mathrm{~m}$ \\
\hline 3 & $h_{3}$ & $0.3 m$ \\
\hline 4 & $l_{1}$ & $0.5 m$ \\
\hline 5 & $l_{2}$ & $0.4 m$ \\
\hline 6 & $m_{1}$ & $3.5 \mathrm{Kg}$ \\
\hline 7 & $m_{2}$ & $2 K g$ \\
\hline 8 & $m_{3}$ & $2.25 \mathrm{Kg}$ \\
\hline 9 & $I_{1}$ & $0.55 \mathrm{Kg} \mathrm{m}^{2}$ \\
\hline 10 & $I_{2}$ & $0.12 \mathrm{Kg} \mathrm{m}^{2}$ \\
\hline 11 & $I_{3}$ & $0.65 \mathrm{Kg} \mathrm{m}^{2}$ \\
\hline 12 & $C_{1}$ & $0.07 \mathrm{Nms}$ \\
\hline 13 & $C_{2}$ & $0.03 \mathrm{Nms}$ \\
\hline 14 & $C_{3}$ & $0.009 \mathrm{Nms}$ \\
\hline
\end{tabular}

Figure 3 Block-diagram of the pendulum system

Table 2 Nominal values of the parameters

\begin{tabular}{|l|l|l|}
\hline No & Symbol & Value \\
\hline
\end{tabular}

The state space representation of the triple inverted pendulum becomes

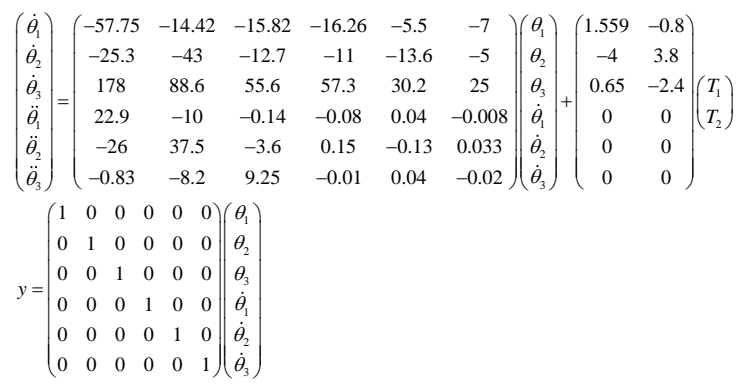

\section{The Proposed Controllers Design 3.1 LQR Controller Design}

The principle of most reliable optimal control is involved with working a dynamic system at minimum cost. The case wherein the system dynamics are described via a fixed of linear differential equations and the cost is defined through a quadratic function is referred to as the LQ problem. One of the primary outcomes within the theory is that the solution is furnished with the aid of the linear quadratic regulator (LQR). The block diagram of the triple inverted pendulum with LQR controller is shown below in Figure 4. 


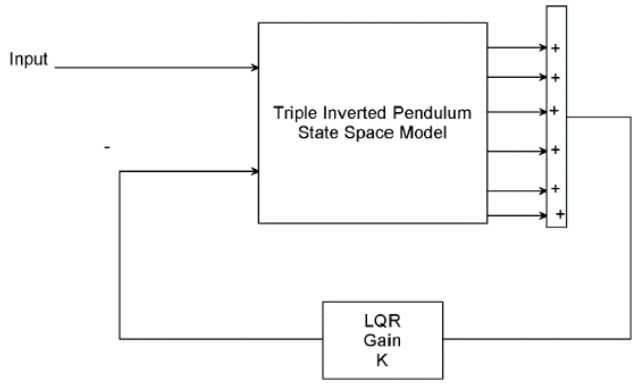

Figure 4 Block diagram of the triple inverted pendulum with LQR controller

In this paper, the value of $\mathrm{Q}$ and $\mathrm{R}$ is chosen as

$$
Q=10\left(\begin{array}{llllll}
1 & 0 & 0 & 0 & 0 & 0 \\
0 & 1 & 0 & 0 & 0 & 0 \\
0 & 0 & 1 & 0 & 0 & 0 \\
0 & 0 & 0 & 1 & 0 & 0 \\
0 & 0 & 0 & 0 & 1 & 0 \\
0 & 0 & 0 & 0 & 0 & 1
\end{array}\right) \text { and } R=\left(\begin{array}{ll}
5 & 0 \\
0 & 5
\end{array}\right)
$$

The value of obtained feedback gain matrix $\mathrm{K}$ of LQR is given by

$$
K=\left(\begin{array}{llllll}
87.4053 & 32.8355 & 25.6454 & 27.1508 & 11.2981 & 11.1817 \\
97.7657 & 45.7910 & 30.0834 & 31.2118 & 15.6479 & 12.9896
\end{array}\right)
$$

\subsection{Pole Placement Controller Design}

Pole placement, is a way employed in feedback control system principle to region the closed-loop poles of a plant in predecided locations in the s-plane. Placing poles is proper because the region of the poles corresponds immediately to the eigenvalues of the system, which control the traits of the reaction of the system. The system ought to be considered controllable on the way to put into effect this technique. The block diagram of the triple inverted pendulum with pole placement controller is shown in Figure 5.

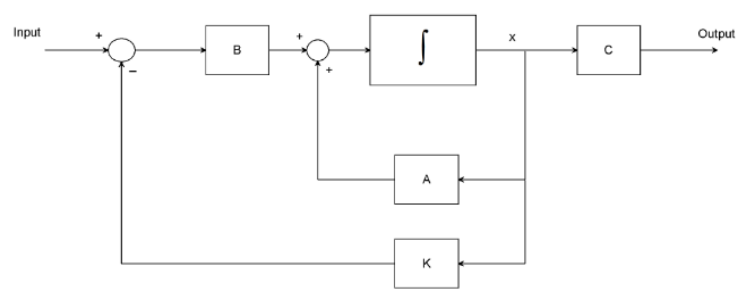

Figure 5 Block diagram of the triple inverted pendulum with pole placement controller

The state equations for the closed-loop system of Figure 5 can be written by inspection as

$$
\begin{aligned}
& \dot{x}=A x+B u=A x+B(-K x)=(A-B K) x \\
& y=C x
\end{aligned}
$$

The poles for this system is chosen as

$$
P=[-1,-2,-3,-4,-5,-6]
$$

Solving using Matlab the robust pole placement algorithm gain will be

$$
K=\left[\begin{array}{rrrrrr}
19329 & 8885 & 7472 & 11601 & 5861 & 6699 \\
23483 & 10820 & 9086 & 14362 & 7268 & 8307
\end{array}\right]
$$

\section{Result and Discussion \\ 4.1 Controllability and Observability of the Pendulum}

A system (state space representation) is controllable iff the controllable matrix $\mathrm{C}=[\mathrm{B}$ $\mathrm{AB} A 2 \mathrm{~B}$....An-1B] has rank $n$ where $n$ is the number of degrees of freedom of the system.

In our system, the controllable matrix $\mathrm{C}=[\mathrm{B}$ AB A2B A3B A4B A5B] has rank 6 which the degree of freedom of the system. So, the system is controllable.

A system (state space representation) is Observable iff the Observable matrix $\mathrm{D}=[\mathrm{C}$ CA CA2....CAn-1] T has a full rank $n$. 
In our system, the Observable matrix $\mathrm{D}=[\mathrm{C}$ CA CA2 CA3 CA4 CA5] T has a full rank of 6. So, the system is Observable.

\subsection{Open Loop Impulse Response of the Triple Inverted Pendulum}

The open loop simulation for a $1 \mathrm{Nm}$ impulse input of torque 1 for angular displacement 1 , 2 and 3 and for angular velocity 1, 2 and 3 is shown in Figure 6, 7, 8, 9, 10 and 11 and for torque 2 input the angular displacement 1, 2 and 3 and for angular velocity 1, 2 and 3 is shown in Figure 12, 13, 14, 15, 16 and 17 respectively.

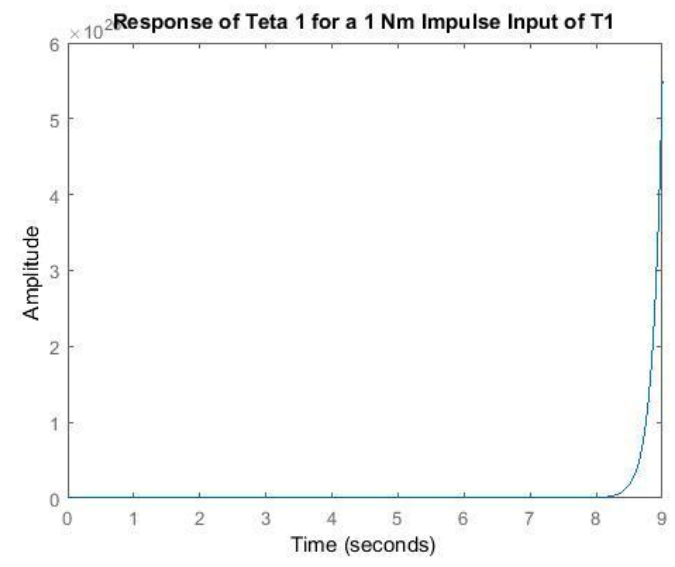

Figure 6 Response of Teta 1

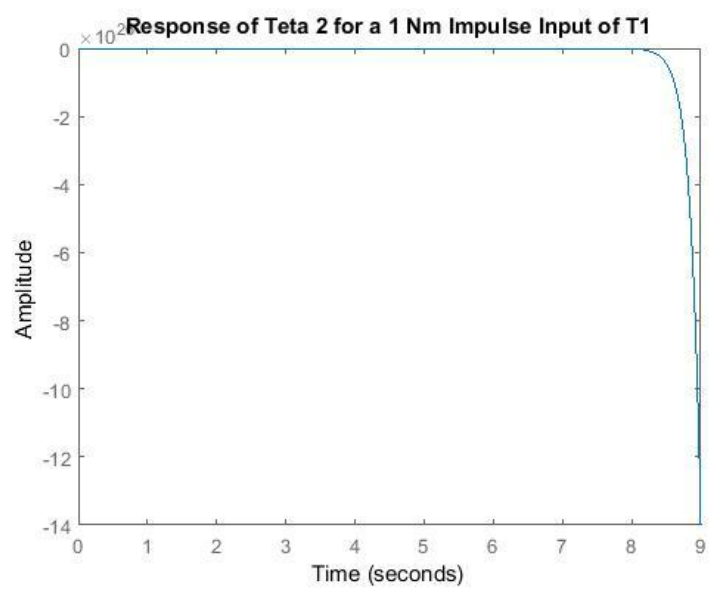

Figure 7 Response of Teta 2

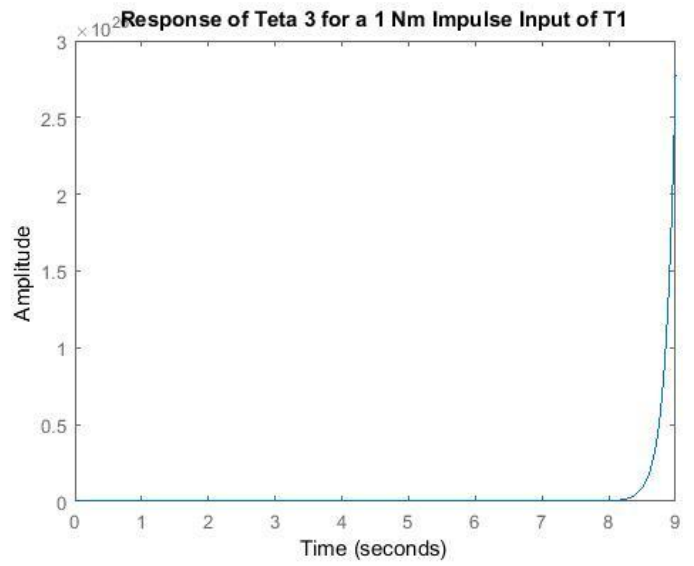

Figure 8 Response of Teta 3

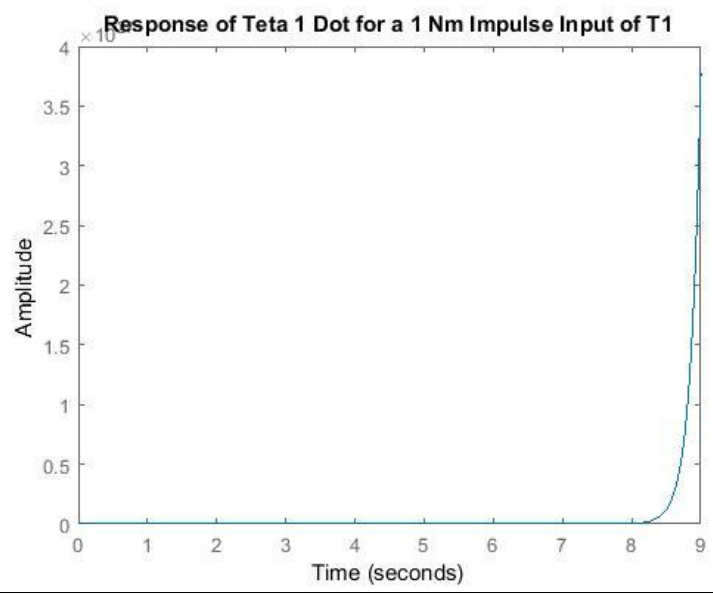

Figure 9 Response of Teta 1 Dot

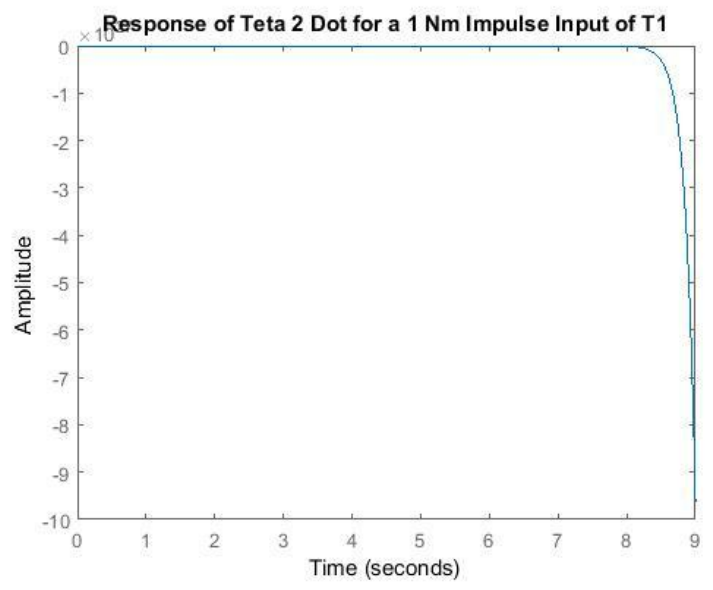

Figure 10 Response of Teta 2 Dot 


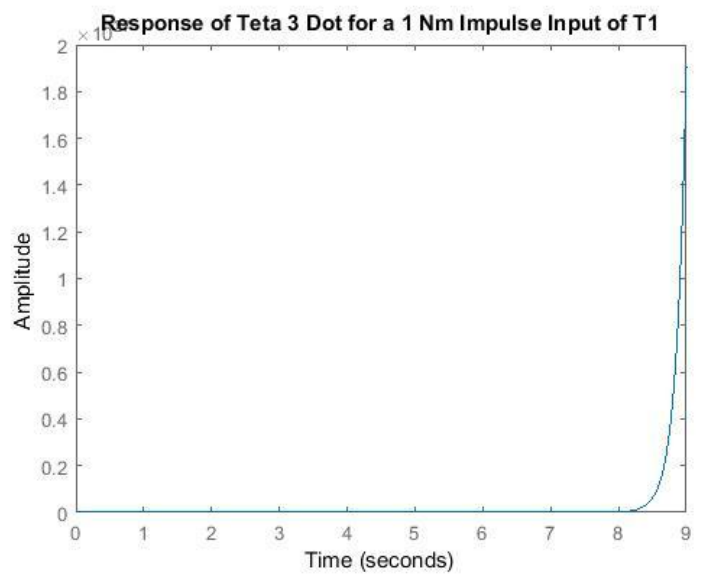

Figure 11 Response of Teta 3 Dot

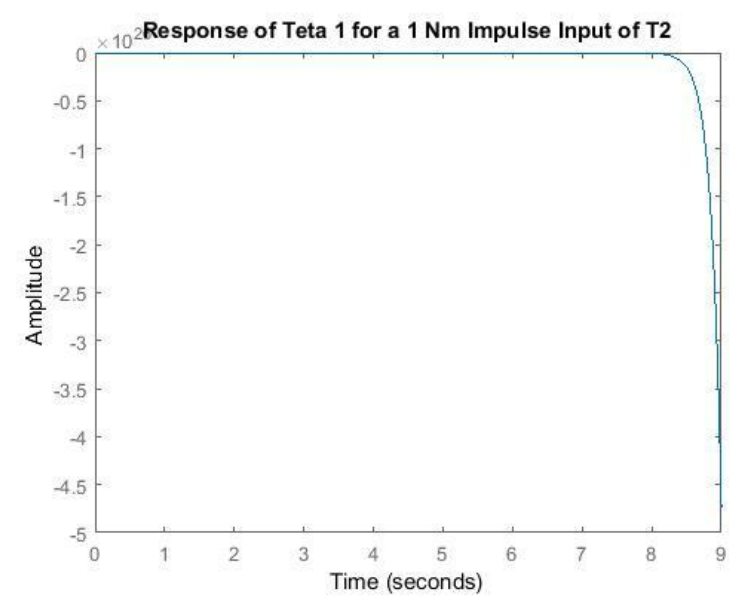

Figure 12 Response of Teta 1

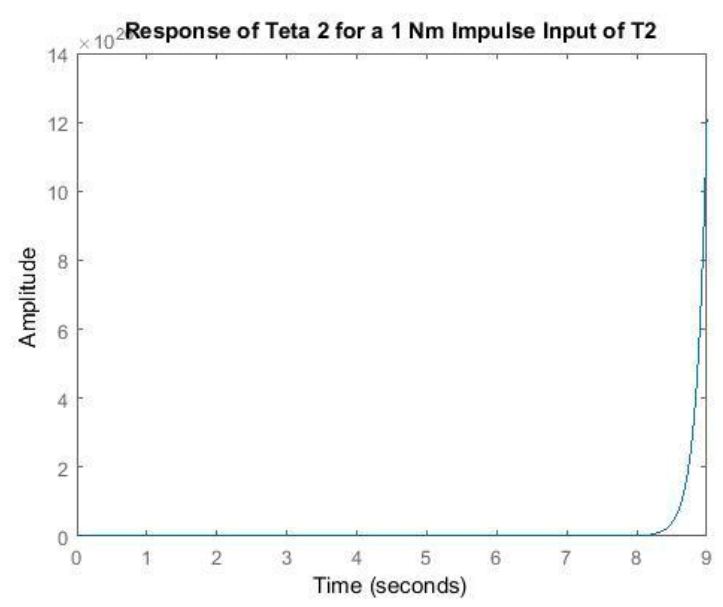

Figure 13 Response of Teta 2

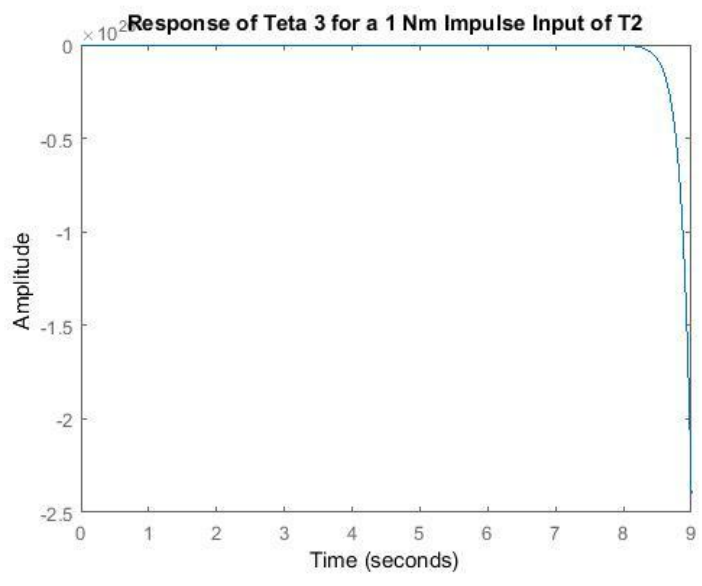

Figure 14 Response of Teta 3

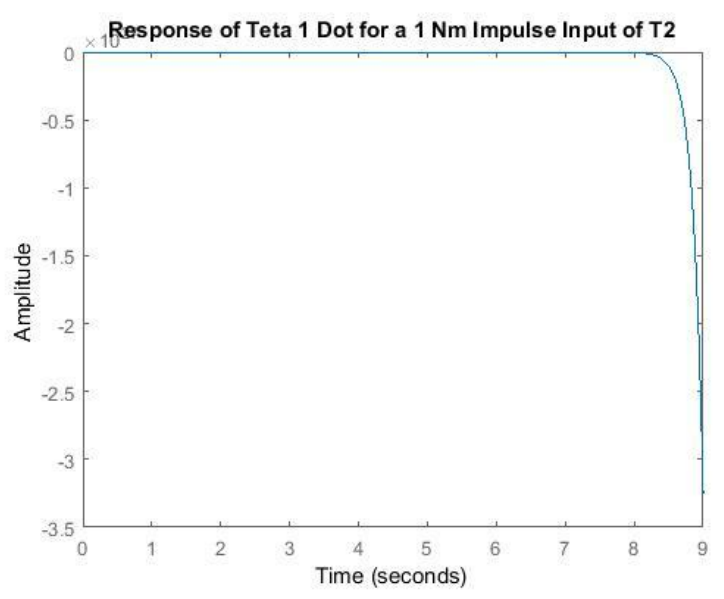

Figure 15 Response of Teta 1 Dot

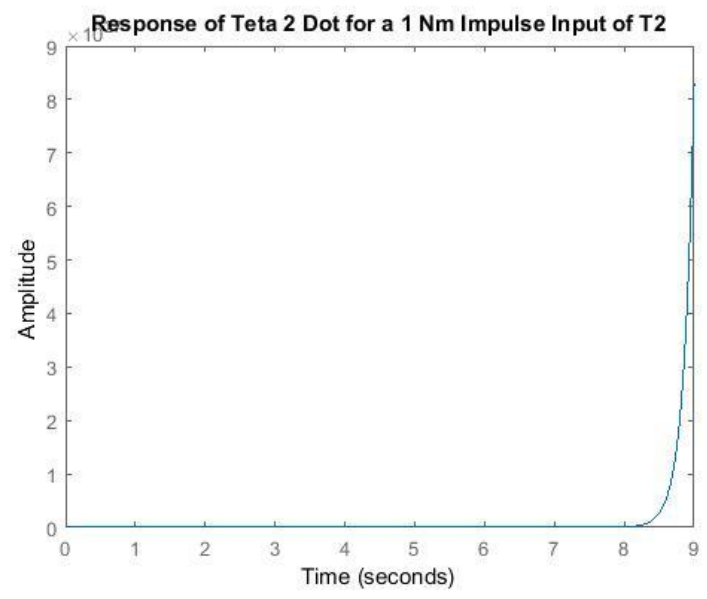

Figure 16 Response of Teta 2 Dot 


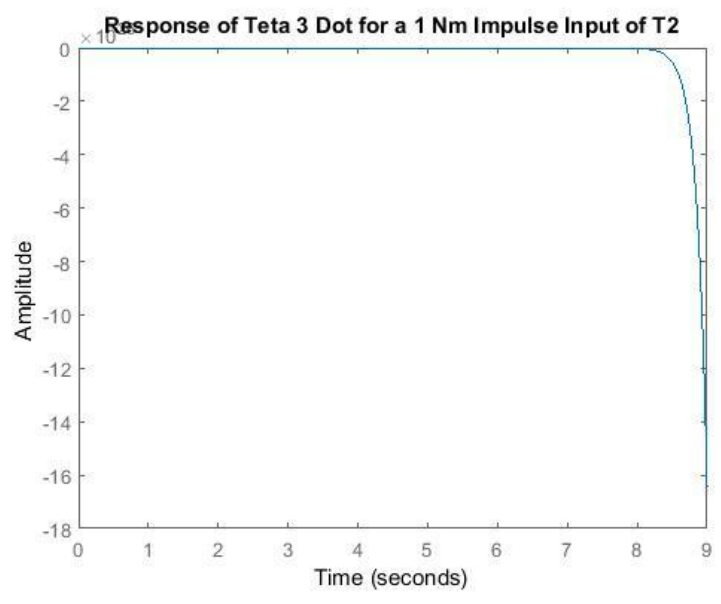

Figure 17 Response of Teta 3 Dot

As we seen from the Figures above the angular displacements and the angular velocities are unstable.

\subsection{Comparison of the Triple Inverted Pendulum with LQR and Pole Placement Controllers for Impulse Input Signal}

The comparison of the triple inverted pendulum with LQR and pole placement controller for a $1 \mathrm{Nm}$ impulse input of torque 1 for angular displacement 1,2 and 3 and for angular velocity 1,2 and 3 is shown in Figure $18,19,20,21,22$ and 23 and for torque 2 input the angular displacement 1, 2 and 3 and for angular velocity 1, 2 and 3 is shown in Figure 24, 25, 26, 27, 28 and 29 respectively.

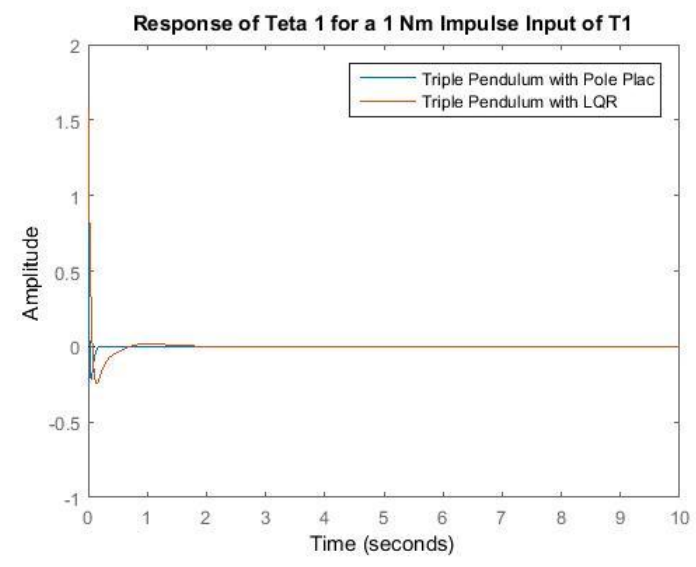

Figure 18 Response of Teta 1

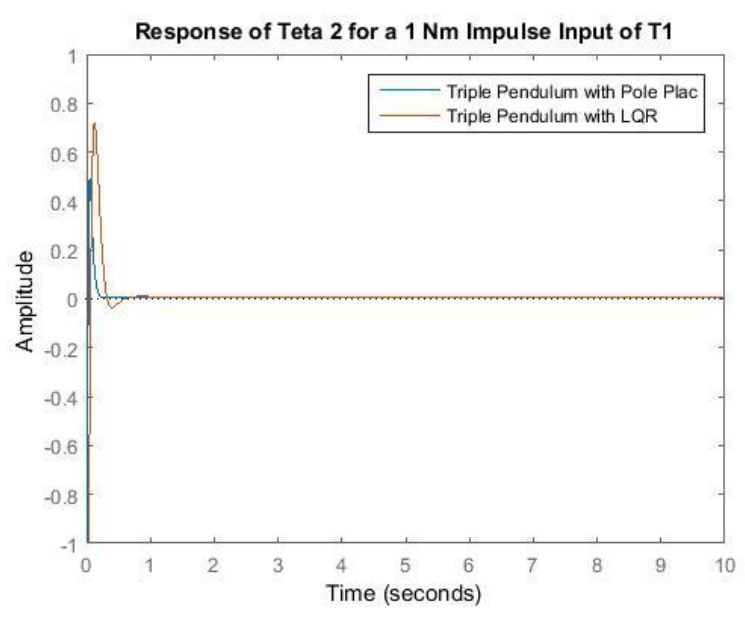

Figure 19 Response of Teta 2

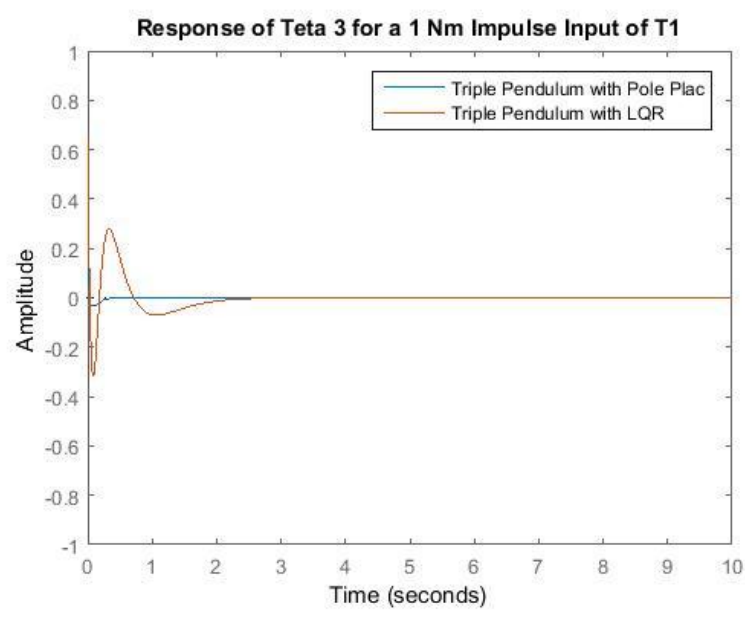

Figure 20 Response of Teta 3

As we seen from Figure 18, 19 and 20, for the impulse signal the angles starts to increase and returns to zero degree for the two controllers but the pendulum with LQR controller has a high overshoot with more settling time than the pendulum with pole placement controller. 


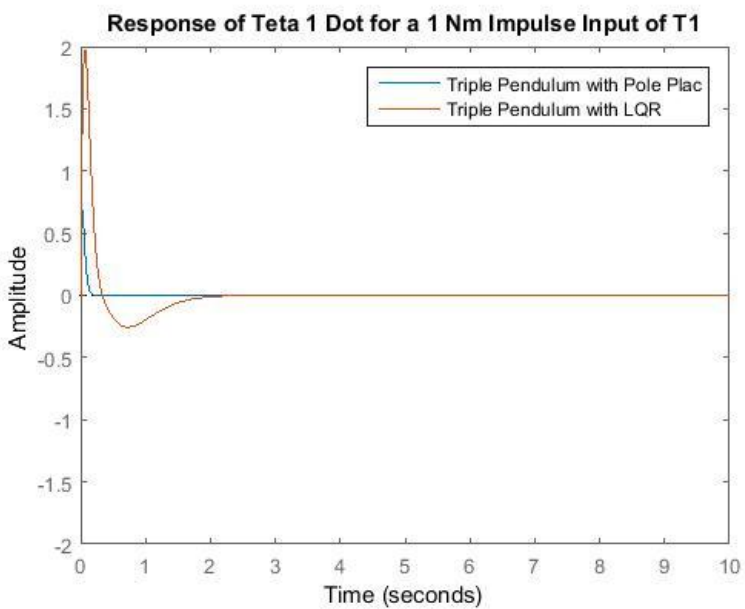

Figure 21 Response of Teta 1 Dot

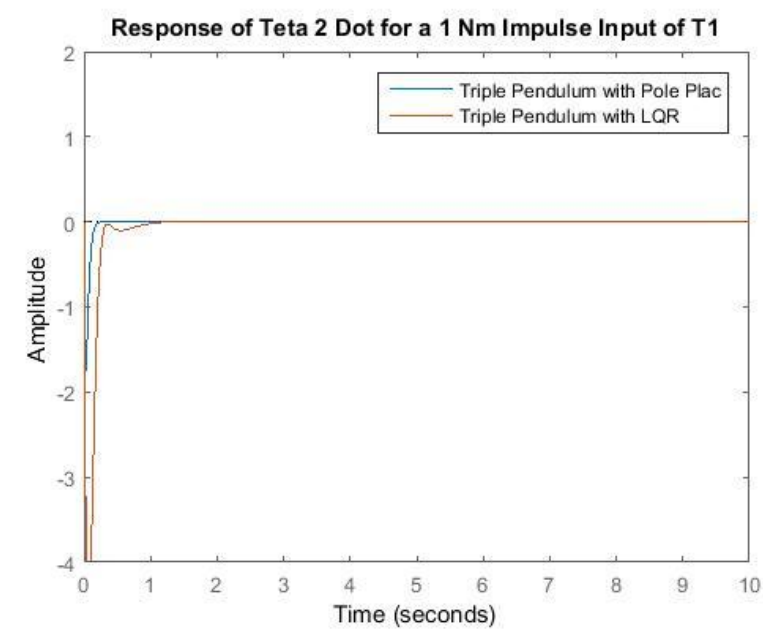

Figure 22 Response of Teta 2 Dot

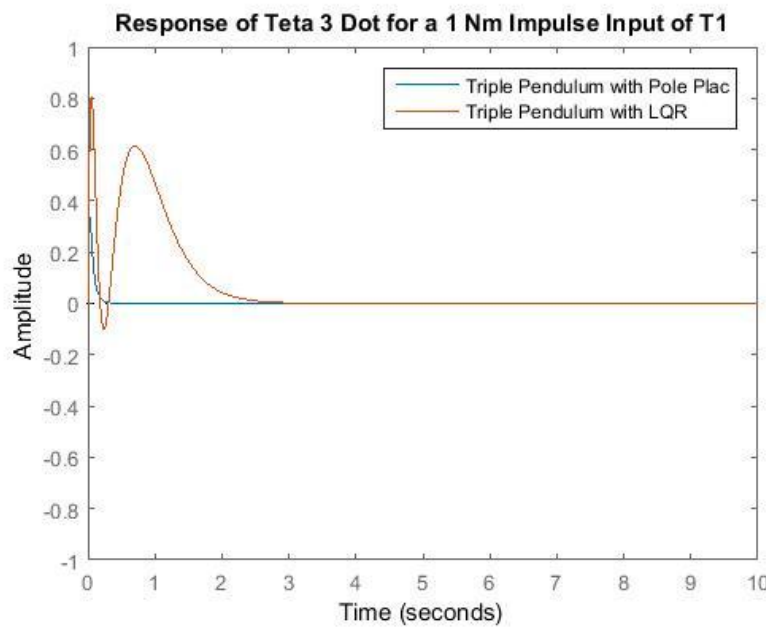

Figure 23 Response of Teta 3 Dot As we seen from Figure 21, 22 and 23, for the impulse signal the angular velocities starts to increase and returns to zero for the two controllers but the pendulum with LQR controller has a high overshoot with more settling time than the pendulum with pole placement controller.

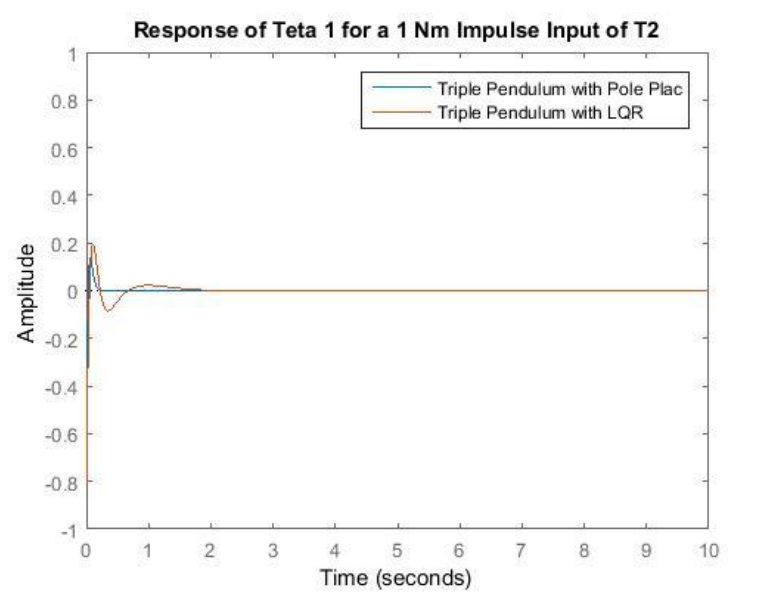

Figure 24 Response of Teta 1

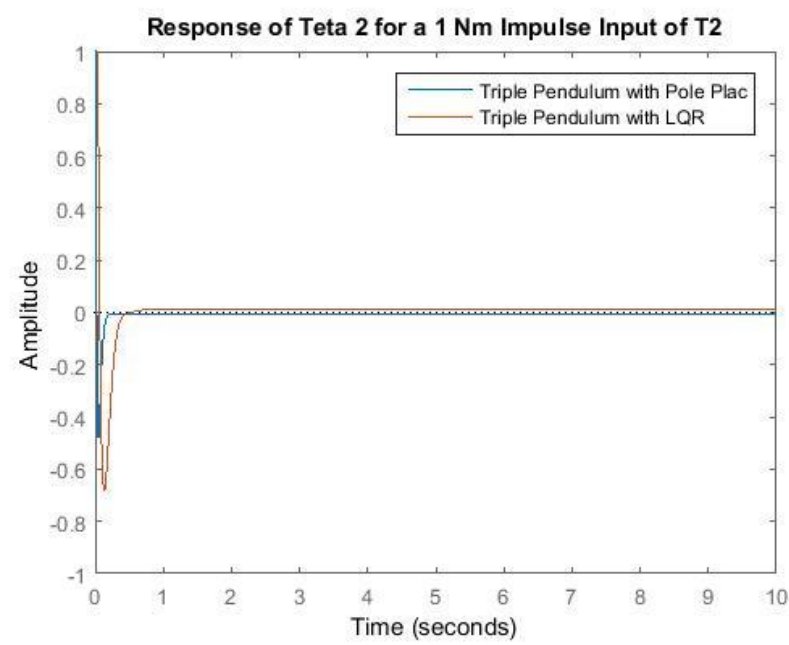

Figure 25 Response of Teta 2 


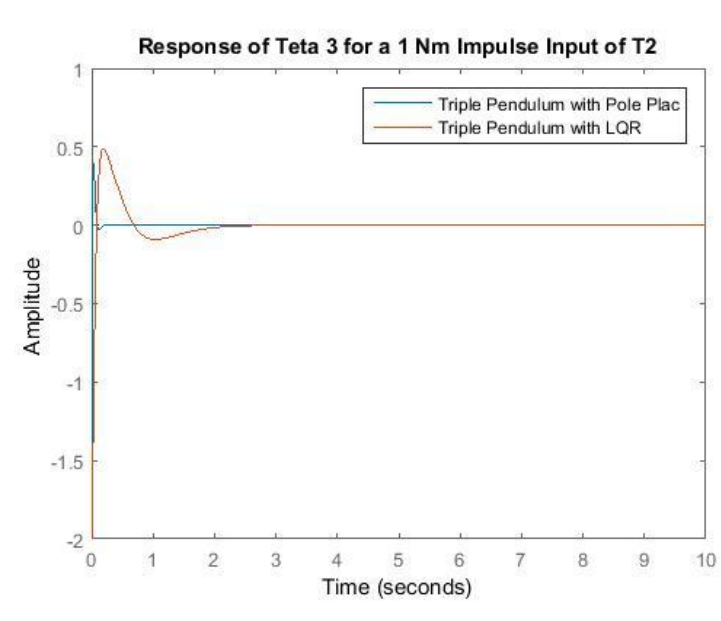

Figure 26 Response of Teta 3

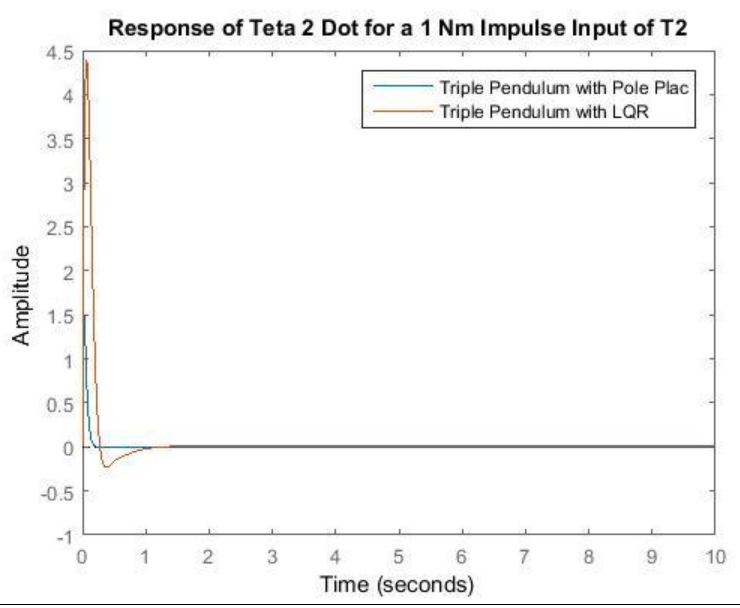

Figure 28 Response of Teta 2 Dot

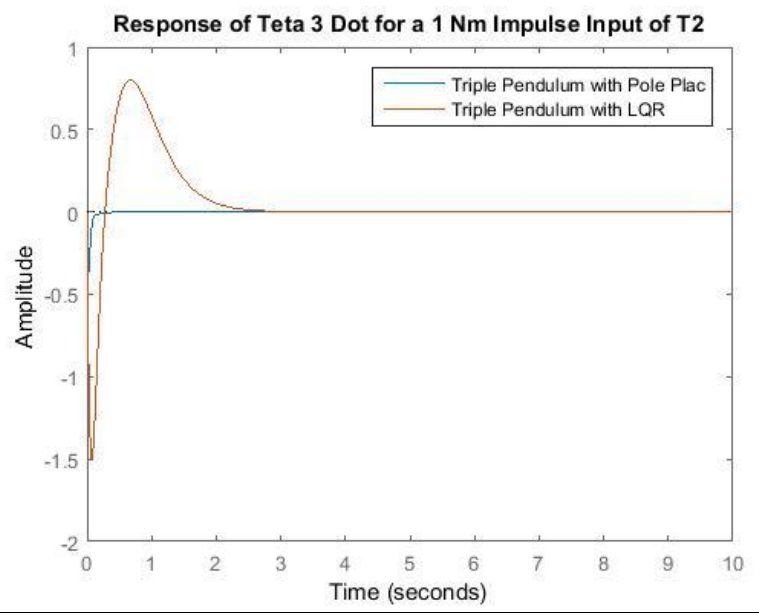

Figure 29 Response of Teta 3 Dot

As we seen from Figure 27, 28 and 29, for the impulse signal the angular velocities starts to increase and returns to zero for the two controllers but the pendulum with LQR controller has a high overshoot with more settling time than the pendulum with pole placement controller.

\section{Conclusion}

In this paper, stabilization of the triple inverted pendulum with LQR and pole placement controller have been analyzed simulated and compared suceesfully. The 
open loop simulation prove that the system is not stable without feedback control system. Comparison of the proposed controllers for an impulse input have been done and the system with pole placement controller improves the stability of the system.

\section{Reference}

[1]. Mustefa Jibril et al. "Robust Control Theory Based Performance Investigation of an Inverted Pendulum System using Simulink" International Journal of Advance Research and Innovative Ideas in Education, Vol. 6, Issue 2, pp. 808814, 2020.

[2].Ali Rohan et al. "Design of Fuzzy Logic Based Controller for Gyroscopic Inverted Pendulum System" Int. J. Fuzzy Log. Intell. Syst, Vol. 18, Issue 1, pp. 58-64, 2018.

[3].Xiaoping H. et al. "Optimization of Triple Inverted Pendulum Control Process Based on Motion Vision" EURASIP Journal on Image and Video Processing, No. 73, 2018.

[4]. R. Dimas P. et al. "Implementation of Push Recovery Strategy using Triple Linear Inverted Pendulum Model in T-Flow Humanoid Robot" Journal of Physics: Conference Series, Vol. 1007, 2018.

[5]. Wei Chen et al. "Simulation of a Triple Inverted Pendulum Based on Fuzzy Control” WJET, Vol. 4, No. 2, 2016.

[6].Tang $\mathrm{Y}$ et al. "A New Fuzzy Evidential Controller for Stabilization of the Planar Inverted Pendulum System" PLOS ONE, Vol. 11, Issue 8, 2016.
[7]. Yuanhong D. et al. "Multi Mode Control Based on HSIC for Double Pendulum Robot" Journal of Vibroengineering, Vol. 17, Issue 7, pp. 3683-3692, 2015. 